\title{
Lower limb events in individuals with type 2 diabetes: evidence for an increased risk associated with diuretic use
}

\author{
Louis Potier ${ }^{1,2,3} \cdot$ Ronan Roussel ${ }^{1,2,3}$. Gilberto Velho ${ }^{3}$ Pierre-Jean Saulnier ${ }^{4,5,6}$ • Anisoara Bumbu ${ }^{1}$. Odette Matar ${ }^{1}$. \\ Fabrice Schneider ${ }^{4,5,6,7} \cdot$ Stéphanie Ragot ${ }^{4,5,6} \cdot$ Michel Marre $^{1,2,3,8} \cdot$ Kamel Mohammedi $^{9,10,11} \cdot$ Samy Hadjadj $^{12}$
}

Received: 19 September 2018 / Accepted: 29 January 2019 / Published online: 26 February 2019

(C) Springer-Verlag GmbH Germany, part of Springer Nature 2019

\begin{abstract}
Aims/hypothesis Recently, safety data signalled an increased risk of amputations in people taking canagliflozin, a sodium -glucose cotransporter 2 (SGLT2) inhibitor. If this side effect is due to drug-induced hypovolaemia, diuretics should also increase that risk. The aim of this study was to analyse the association between diuretic use and the risk of lower limb events (LLEs) in people with type 2 diabetes.

Methods SURDIAGENE (SUivi Rénal, DIAbète de type 2 et GENEtique) is a prospective observational cohort that includes people with type 2 diabetes enrolled from 2002 to 2012 and followed-up until onset of LLE, death or 31 December 2015, whichever came first. Primary outcome was the first occurrence of LLE, a composite of lower limb amputation (LLA) and lower limb revascularisation (LLR). The rates of primary outcome were compared between participants taking and not taking diuretics at baseline in a Cox-adjusted model.

Results At baseline, of the 1459 participants included, 670 were taking diuretics. In participants with and without diuretics, the mean ages were 67.1 and 62.9 years and $55.8 \%$ and $59.8 \%$ were men, respectively. During a median follow-up of 7.1 years, the incidence of LLE was 1.80 per 100 patient-years in diuretic users vs 1.00 in non-users $(p<0.001)$. The HR for LLE in users vs non-users was 2.08 (95\% CI 1.49, 2.93), $p<0.001$. This association remained significant in a multivariable-adjusted model (1.49 $[1.01,2.19] ; p=0.04$ ) and similar after considering death as a competing risk (subhazard ratio $1.89[1.35,2.64] ; p<0.001$ ). When separated, LLA but not LLR, was associated with the use of diuretics: $2.01(1.14,3.54), p=0.02$ and $1.05(0.67,1.64), p=0.84$, respectively, in the multivariable-adjusted model.

Conclusions/interpretation Among people with type 2 diabetes treated with diuretics, there was a significant increase in the risk of LLE, predominantly in the risk of LLA.
\end{abstract}

Keywords Diuretics $\cdot$ Lower limb amputation $\cdot$ Lower limb revascularisation $\cdot$ Type 2 diabetes

Electronic supplementary material The online version of this article (https://doi.org/10.1007/s00125-019-4835-z) contains peer-reviewed but unedited supplementary material, which is available to authorised users.

Louis Potier

louis.potier@gmail.com

1 Department of Diabetology, Endocrinology and Nutrition, Bichat Hospital, Assistance Publique - Hôpitaux de Paris, DHU FIRE, 46 rue Henri Huchard 75877, Paris Cedex 18, France

2 UFR de Médecine, Paris Diderot University, Sorbonne Paris Cité, Paris, France

3 Centre de Recherche des Cordeliers, INSERM, Sorbonne Université, USPC, Université Paris Descartes, Université Paris Diderot, Paris, France

4 CIC1402, INSERM, Poitiers, France
5 UFR de Médecine et Pharmacie, Poitiers University, Poitiers, France

6 Clinical Investigation Centre, CHU de Poitiers, Poitiers, France

7 Department of Vascular Surgery, Centre Hospitalier Universitaire de Poitiers, Poitiers, France

8 Fondation Ophtalmologique Adolphe de Rothschild, Paris, France

9 Department of Diabetology, Endocrinology and Nutrition, Hôpital Haut-Lévêque, Bordeaux, France

10 Bordeaux University, Bordeaux, France

11 Inserm U1219 'Bordeaux Population Health', Bordeaux, France

12 Institut du Thorax, Inserm, CNRS, CHU Nantes, Nantes, France 


\section{Research in context}

\section{What is already known about this subject?}

- Lower limb amputation and revascularisation are highly prevalent in people with type 2 diabetes

- Some evidence from randomised controlled trial and observational data suggests that sodium-glucose cotransporter 2 (SGLT2) inhibitors could be associated with an increased risk of lower limb amputation

- A potential mechanism for the increased risk of lower limb amputation observed with SGLT2 inhibitors is glycosuriainduced osmotic diuresis

What is the key question?

- Is there an association between diuretic use and lower limb events in people with type 2 diabetes?

\section{What are the new findings?}

- In a single-centre prospective observational cohort of individuals with type 2 diabetes, diuretic use was associated with a higher risk of lower limb amputation

\section{How might this impact on clinical practice in the foreseeable future?}

- The clinical implications may be that diuresis-inducing drugs should be used with caution and blood volume in people with diabetes and peripheral arterial disease or foot ulcers should be carefully assessed

ACR

CANVAS

eGFR

LLA

LLE

LLR

PAD

RAAS

SGLT2

$\begin{array}{ll}\text { Abbreviations } & \\ \text { ACR } & \text { Albumin/creatinine ratio } \\ \text { CANVAS } & \begin{array}{l}\text { Canagliflozin Cardiovascular Assessment } \\ \text { Study }\end{array} \\ \text { eGFR } & \text { Estimated GFR } \\ \text { LLA } & \text { Lower limb amputation } \\ \text { LLE } & \text { Lower limb event } \\ \text { LLR } & \text { Lower limb revascularisation } \\ \text { PAD } & \text { Peripheral arterial disease } \\ \text { RAAS } & \text { Renin-angiotensin-aldosterone system } \\ \text { SGLT2 } & \text { Sodium-glucose cotransporter 2 } \\ \text { SURDIAGENE } & \text { SUivi Rénal, DIAbète de type 2 et } \\ & \text { GENEtique }\end{array}$

Abbreviations

In the Canagliflozin Cardiovascular Assessment Study (CANVAS), the use of canagliflozin, compared with placebo, has been associated with doubling of the risk of LLA [6]. Canagliflozin, a sodium-glucose cotransporter 2 (SGLT2) inhibitor, is a glucose-lowering agent with a mild diuretic action consequent to glycosuria-induced osmotic diuresis. A potential mechanism for the increased risk of LLA in CANVAS could be this drug-induced hypovolaemia. With this hypothesis, diuretics should also show a similar safety profile. A number of studies have analysed the association between diuretic use and LLA but were limited regarding type of diuretic or number of participants with diabetes [7, 8].

In this study, we analysed the association between diuretics and the incidence of lower limb events (LLEs), LLAs or lower limb revascularisations (LLRs), in a prospective observational cohort of people with type 2 diabetes.

\section{Methods}

Diabetes mellitus is the leading cause of lower limb amputation (LLA) [1]. The high prevalence of this debilitating complication in people with diabetes is explained by a wide range of factors, such peripheral arterial disease (PAD), diabetic neuropathy, impaired wound healing, susceptibility to infection and others [2]. Ischaemia is associated with a dramatic increase risk of LLA in people with diabetes and foot ulcers [3]. Hypovolaemia could further decrease peripheral perfusion in people with PAD, favouring decompensation and eventually LLA. There is evidence from case reports that extracellular volume depletion could lead to lower limb or mesenteric ischaemia $[4,5]$.
Study protocols and participants The SURDIAGENE (SUivi Rénal, DIAbète de type 2 et GENEtique) study is a French prospective single-centre study in which a cohort of people with type 2 diabetes regularly visit the Diabetes Department at Poitiers University Hospital, Poitiers, France [9]. Individuals aged $\geq 18$ years with a definite diagnosis of type 2 diabetes for $\geq 2$ years were recruited from 2002 to 2012 . The outcomes have been updated prospectively every 2 years from 2007 to 2015. The main exclusion criteria were non-diabetic kidney disease and follow-up duration $<1$ month. The Poitiers University Hospital Ethics Committee approved the study 
design (Comité de Protection des Personnes Ouest III). All participants provided written informed consent.

Clinical and biological data, including personal medical history and medication use, were collected at baseline. Use of medication was reported by the physician and data were collected at baseline from drug-prescription analyses. Diuretic classes included thiazide, loop and potassium-sparing diuretics. Mineralocorticoid receptor antagonists were not considered as diuretics in our analysis. No data were available on the type of diuretic used by each participant and diuretic users were assembled in a single group. Date of first treatment initiation was not available for this cohort. A prevalent-user design was employed. Estimated (e)GFR was calculated using the Chronic Kidney Disease Epidemiology Collaboration formula [10].

Clinical outcomes The primary outcome was first occurrence of an LLE during follow-up. LLE was defined as a composite of LLA or LLR, whichever came first. LLA was defined as an amputation at or above the metatarsophalangeal joint. LLR was defined as the requirement of peripheral (aorta or lower limb arteries) revascularisation procedure by angioplasty or bypass. None of the reported amputations was traumatic. Each LLE outcome was considered separately as a secondary endpoint. Outcomes were individually determined from individuals' hospital records, French death certificate registries and interviews with their general practitioners. Those moving out of the hospital area (Poitou-Charentes district) were censored at the time of their departure. An independent adjudication committee reviewed every prospectively collected event [9]. Each endpoint was reviewed by two independent physicians and, in the case of disagreement, the whole committee discussed the endpoint until agreement was reached. Dates of events reported by the adjudication committee were the exact dates of events.

Statistical analyses Continuous variables are expressed as mean \pm SD or as median (25th, 75th percentiles) for those with skewed distribution. Categorical variables are expressed as the number of participants, with the corresponding percentages. Comparisons between users and non-users of diuretics were conducted using the Student's $t$, Wilcoxon or $\chi^{2}$ tests.

Participants with at least one missing value of any of the covariates used in the adjusted Cox model were removed from all analyses that included the covariate.

The probability of remaining free from LLE and individual components of LLE were plotted as Kaplan-Meier curves according to use of diuretic at baseline and compared using the logrank test. Cox proportional hazards regression models were fitted to estimate HRs, with associated $95 \%$ CIs, for endpoints during follow-up for participants taking diuretics compared with participants not taking diuretics. We included in our multivariable Cox model all baseline characteristics with $p<0.10$ in the comparison between participants with incident outcomes during follow-up and those with no events: age; diabetes duration; sex; BMI; systolic BP; hypertension; diabetic retinopathy; previous cardiovascular disease; previous amputation; previous PAD; HDL-cholesterol; albumin/ creatinine ratio; eGFR; antiplatelet/anticoagulant; BPlowering agents; statins; biguanides; and insulin use (data from electronic supplementary material [ESM] Table 1). The high rate of comorbidities in participants prone to LLE meant that a large proportion of participants might have succumbed to death before LLE. Because of this, our analyses were also adjusted for death as a competing risk using the Fine and Gray method [11]. The Schoenfeld residuals method was used to assess the proportional hazards assumption for the association between primary endpoint and diuretic use $(p=0.83)$.

People with heart failure are more likely to be treated with diuretics and this could lead to allocation bias in this study. However, baseline data for heart failure were not available for our cohort. To overcome this limitation, we did a sensitivity analysis in a subgroup of participants without an episode of congestive heart failure during follow-up $(n=1212)$, with the assumption that this group would have a lower prevalence of heart failure at baseline compared with participants experiencing congestive heart failure during follow-up.

We also compared diuretic users with users of an active comparator class, renin-angiotensin-aldosterone system (RAAS) blockers (e.g. angiotensin conversion enzyme inhibitors and angiotensin receptor blockers). The choice of this comparator was based on the number of participants taking only one class for comparison and because these classes have an opposite action on the RAAS, which has been shown to be involved in PAD [12].

Finally, to better account for differences in baseline characteristics of participants taking or not taking diuretics, participants were propensity-score matched (1:1) based on baseline characteristics using a nearest-neighbour matching algorithm and a calliper at 0.1 [13]. Baseline characteristics used for matching included: age; sex; diabetes duration; BMI; smoking; systolic and diastolic BP; history of hypertension; history of cardiovascular disease; history of myocardial infarction; history of LLE; total cholesterol; HDL-cholesterol; triacylglycerols; diabetic retinopathy; albuminuria, $\mathrm{HbA}_{1 c}$; eGFR; antiplatelet or anticoagulant use; BP-lowering drug use; $\beta$ blocker use; RAAS-blocker use; statin use; and insulin use. We matched 964 participants $(71.9 \%$ and $61.1 \%$ of diuretic users and non-users before matching, respectively). After propensity-score matching, all absolute standardised differences were $<10 \%$, indicating robust matching [13]. Cox models were fitted for endpoints with diuretic use as the covariate in the matched cohort.

Statistics were performed with JMP (SAS Institute, Cary, $\mathrm{NC}, \mathrm{USA}$ ) and R software (version 3.4.2). Two-sided $p$ values $<0.05$ were considered significant. 


\section{Results}

Baseline characteristics Among the 1468 participants enrolled in the full cohort, nine participants were excluded for missing baseline data on diuretic use. Characteristics of participants at baseline are shown in Table 1 . Participants taking diuretics at baseline were significantly older, had significantly lower eGFR, higher albumin/ creatinine ratio (ACR), more frequently used other BPlowering medications and more often had history of cardiovascular disease compared with those not using diuretics. LLE occurred in 142 participants $(9.7 \%)$ during follow-up (first event to occur in case of recurrence). During a follow-up of 7.1 years, the incidence rate of LLE was 1.38 per 100 person-years. Taken individually, LLA occurred in 79 (5.4\%) and LLR in 98 (6.7\%) participants. The incidence rates of LLA and LLR were 0.78 and 0.98 person-years, respectively. Participants who experienced either event, compared with those who did not, were more often men, had longer duration of diabetes and had higher rates of complications and previous cardiovascular disease. They had higher rates of previous amputation and PAD and were more often treated with cardioprotective medications (BP-lowering drugs, statins and antiplatelet and anticoagulant agents) (ESM Table 1).

Diuretic use and LLEs during follow-up During a median follow-up of 7.1 years, the incident rate of LLE was 1.80 per 100 patient-years in diuretic users vs 1.00 in non-users $(p<0.001)$. The probabilities of remaining event-free during follow-up according to diuretic use are presented in Fig. 1. Cox proportional hazards survival regression analyses showed significant association between the use of diuretics and the incidence of LLE. After adjustment for confounders, the rate of LLE remained higher in participants using diuretics compared with non-users (Table 2).

Among participants taking or not taking diuretics at baseline, the incidence of LLA was 1.07 vs 0.50 per 100 patientyears, respectively $(p<0.001)$ and for LLR 1.14 vs 0.80 per 100 patient-years, respectively $(p=0.04)$. Differences in the incidence of LLA according to the site of amputation (transfemoral, transtibial or transmetatarsal) were not significant $(p=0.86)$. The rate of LLA remained significantly increased after adjustment whereas the risk of LLR was no longer significant in diuretic users in the adjusted model (Table 2).

All-cause death occurred in 542 participants (37.1\%) during follow-up, including 78 participants with any LLE during follow-up. In competing-risk regression analyses, subhazard ratios for LLE, LLA and LLR were similar to HRs from the Cox model (Table 2), indicating that death was not a competing risk in the association of diuretic use with outcomes.
Sensitivity analyses We calculated the HR in the subgroup of participants without an incident episode of congestive heart failure during follow-up $(n=1212)$. The incidence rate of LLE and LLA remained significantly higher in participants taking diuretics compared with those not taking diuretics. This increased rate was not observed for LLR (ESM Table 2).

We also compared participants taking diuretics but not RAAS blockers $(n=143)$ with participants taking RAAS blockers but not diuretics $(n=393)$. The adjusted HR was 1.84 (95\% CI 0.97, 3.47; $p=0.06$ ) for LLE.

After propensity-score matching, baseline characteristics between groups were better balanced, with no significant differences between diuretic users and non-users (ESM Table 3). In Cox proportional hazards survival regression analyses, we observed a significantly increased rate of the primary outcome in participants using diuretics compared with non-users (Table 3). The risk of LLA was significantly increased with diuretic use while the increase in LLR did not reach statistical significance (Table 3).

\section{Discussion}

In this analysis of 1459 participants with type 2 diabetes, the use of diuretics was strongly and independently associated with an increased risk of LLE and, more specifically, LLA. We observed consistent findings after using multiple adjustment methods for confounding covariates. Previous epidemiological data have already suggested a similar association in individuals with and without diabetes [7, 8]. The absolute risk of LLE is much higher in longstanding diabetes but, to our knowledge, this is the first longitudinal study supporting an association between diuretic use and LLE in type 2 diabetes.

The association between BP-lowering medications and LLA in diabetes has been investigated in 450,000 participants in a cross-sectional study of electronic medical records [7]. In line with our results, the authors reported a positive association between thiazides and LLA compared with other BPlowering drugs. However, the design was cross-sectional and other classes of diuretic (loop and potassium-sparing diuretics) were not included in the analyses. In another crosssectional study of 1000 participants with known PAD, a 1.5fold increased risk of critical limb ischaemia with the use of loop diuretics was reported but no information was available regarding other types of diuretic [8]. Randomised controlled trials testing diuretics would have been the best design to assess causality, but relevant reports did not include precise data on LLE, probably because these events are expected to occur infrequently in this setting. However, the large Antihypertensive and Lipid Lowering Treatment to Prevent Heart Attack Trial (ALLHAT) showed a trend towards a lower rate of peripheral artery disease events with amlodipine vs chlortalidone, although this did not reach statistical 
Table 1 Characteristics of participants at baseline according to diuretic use

\begin{tabular}{|c|c|c|c|c|}
\hline Characteristic & No diuretic & Diuretic & $p$ & Missing data $n(\%)$ \\
\hline$n$ & 789 & 670 & & \\
\hline Age, years & $62.9 \pm 11.1$ & $67.1 \pm 9.5$ & $<0.001$ & 0 \\
\hline Diabetes duration, years & $13.1 \pm 9.5$ & $16.2 \pm 10.2$ & $<0.001$ & $2(0.1)$ \\
\hline Sex (male), $n(\%)$ & $468(59.3)$ & $374(55.8)$ & 0.20 & 0 \\
\hline BMI, $\mathrm{kg} / \mathrm{m}^{2}$ & $30.7 \pm 6.1$ & $32.0 \pm 6.4$ & $<0.001$ & 0 \\
\hline Current smokers, $n(\%)$ & $99(12.5)$ & $54(8.1)$ & 0.02 & $18(1.0)$ \\
\hline Heart rate, bpm & $71.6 \pm 13.7$ & $70.4 \pm 14.0$ & 0.12 & $8(0.5)$ \\
\hline Systolic BP, mmHg & $131.9 \pm 17.1$ & $133.2 \pm 18.5$ & 0.16 & $7(0.5)$ \\
\hline Diastolic BP, mmHg & $72.8 \pm 10.9$ & $71.9 \pm 11.4$ & 0.12 & $7(0.5)$ \\
\hline Hypertension, $n(\%)$ & $540(68.4)$ & $668(99.7)$ & $<0.001$ & 0 \\
\hline Renal replacement therapy, $n(\%)$ & $12(1.5)$ & $9(1.3)$ & 0.95 & 0 \\
\hline Diabetic retinopathy, $n(\%)$ & $328(41.6)$ & $311(46.4)$ & 0.07 & $6(0.4)$ \\
\hline Previous cardiovascular disease, $n(\%)$ & $185(23.4)$ & $218(32.5)$ & $<0.001$ & 0 \\
\hline Myocardial infarction & $102(12.9)$ & $122(18.2)$ & 0.01 & 0 \\
\hline Coronary revascularisation & $98(12.4)$ & $121(18.1)$ & 0.003 & 0 \\
\hline Stroke & $43(5.4)$ & $40(6.0)$ & 0.75 & 0 \\
\hline Carotid revascularisation & $14(1.8)$ & $21(3.1)$ & 0.13 & 0 \\
\hline PAD & $33(4.2)$ & $40(6.0)$ & 0.15 & 0 \\
\hline History of amputation, $n(\%)$ & $31(3.9)$ & $42(6.3)$ & 0.06 & 0 \\
\hline \multicolumn{5}{|l|}{ Amputation level, $n(\%)$} \\
\hline Toe & $16(2.0)$ & $23(3.4)$ & & 0 \\
\hline Transmetatarsal & $8(1.0)$ & $7(1.0)$ & & 0 \\
\hline Transtibial & $5(0.6)$ & $3(0.4)$ & & 0 \\
\hline Transfemoral & $2(0.3)$ & $9(1.3)$ & & 0 \\
\hline $\mathrm{HbA}_{1 \mathrm{c}}, \mathrm{mmol} / \mathrm{mol}$ & $61.9 \pm 17.5$ & $61.1 \pm 16.1$ & 0.40 & $1(0.06)$ \\
\hline $\mathrm{HbA}_{1 \mathrm{c}}, \%$ & $7.8 \pm 1.6$ & $7.7 \pm 1.5$ & 0.40 & $1(0.06)$ \\
\hline Total cholesterol, mmol/1 & $4.9 \pm 1.1$ & $4.7 \pm 1.2$ & 0.002 & 0 \\
\hline LDL-cholesterol, mmol/1 & $2.8 \pm 0.9$ & $2.6 \pm 1.0$ & $<0.001$ & $59(4.0)$ \\
\hline HDL-cholesterol, mmol/1 & $1.2 \pm 0.4$ & $1.2 \pm 0.4$ & 0.35 & $8(0.5)$ \\
\hline Triacylglycerols, $\mathrm{mmol} / \mathrm{l}^{\mathrm{a}}$ & $1.6(1.1,2.2)$ & $1.6(1.2,2.4)$ & 0.07 & $5(0.3)$ \\
\hline $\mathrm{ACR}, \mathrm{mg} / \mathrm{mmol}^{\mathrm{a}}$ & $2.4(0.9,9.9)$ & $4.1(1.2,19.2)$ & $<0.001$ & $15(1.0)$ \\
\hline 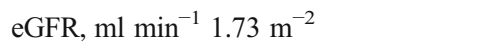 & $77.6 \pm 23.9$ & $66.5 \pm 25.2$ & $<0.001$ & 0 \\
\hline Antiplatelet or anticoagulant agent, $n(\%)$ & $309(39.2)$ & $308(46.0)$ & 0.01 & 0 \\
\hline BP-lowering treatments, $n(\%)$ & $539(68.3)$ & $670(100.0)$ & $<0.001$ & 0 \\
\hline RAAS blockers, $n(\%)$ & $393(49.8)$ & $527(78.7)$ & $<0.001$ & 0 \\
\hline Calcium channel blockers, $n(\%)$ & $201(25.5)$ & $262(39.1)$ & $<0.001$ & 0 \\
\hline$\beta$-blockers, $n(\%)$ & $203(25.7)$ & $296(44.2)$ & $<0.001$ & 0 \\
\hline Statins, $n(\%)$ & $312(39.5)$ & $353(52.7)$ & $<0.001$ & 0 \\
\hline Biguanides, $n(\%)$ & $396(50.2)$ & $286(42.7)$ & 0.01 & 0 \\
\hline Sulfonylureas, $n(\%)$ & $338(42.8)$ & $244(36.4)$ & 0.02 & 0 \\
\hline Thiazolidinediones, $n(\%)$ & $11(1.4)$ & $6(0.9)$ & 0.52 & 0 \\
\hline$\alpha$-Glucosidase inhibitors, $n(\%)$ & $52(6.6)$ & $28(4.2)$ & 0.06 & 0 \\
\hline Insulin, $n(\%)$ & $455(57.7)$ & $425(63.4)$ & 0.03 & 0 \\
\hline
\end{tabular}

Data are expressed as mean $\pm \mathrm{SD}$, with exceptions as noted in footnote a. Quantitative characteristics were analysed with Student's $t$ test on log-transformed data, with the exceptions of those expressed as a median, which were analysed with the Wilcoxon test

Hypertension was defined as systolic BP > $140 \mathrm{mmHg}$ and/or diastolic BP > $90 \mathrm{mmHg}$ or presence of BPlowering medication and history of hypertension

${ }^{\text {a }}$ Expressed as median (25th, 75th percentiles) 


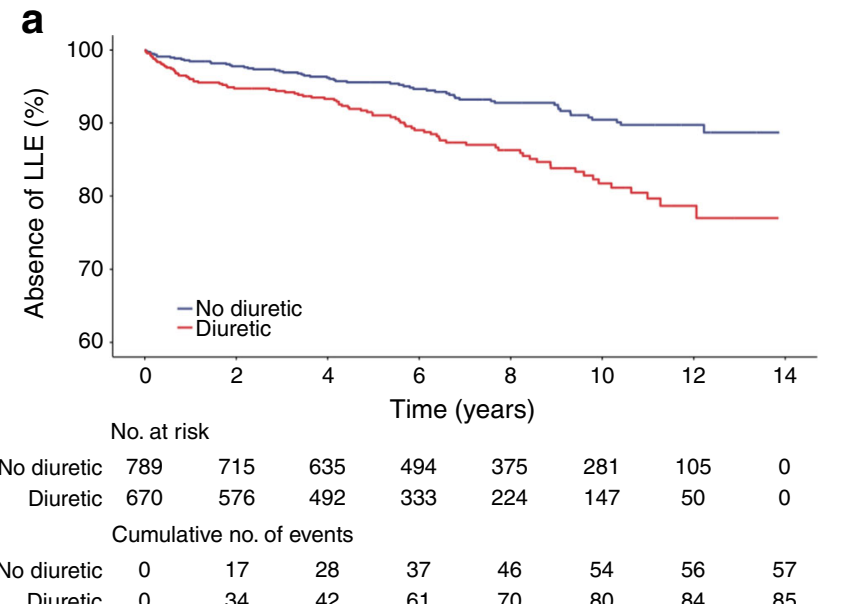

$\begin{array}{rllllllll}\text { No diuretic } & 0 & 17 & 28 & 37 & 46 & 54 & 56 & 57 \\ \text { Diuretic } & 0 & 34 & 42 & 61 & 70 & 80 & 84 & 85\end{array}$
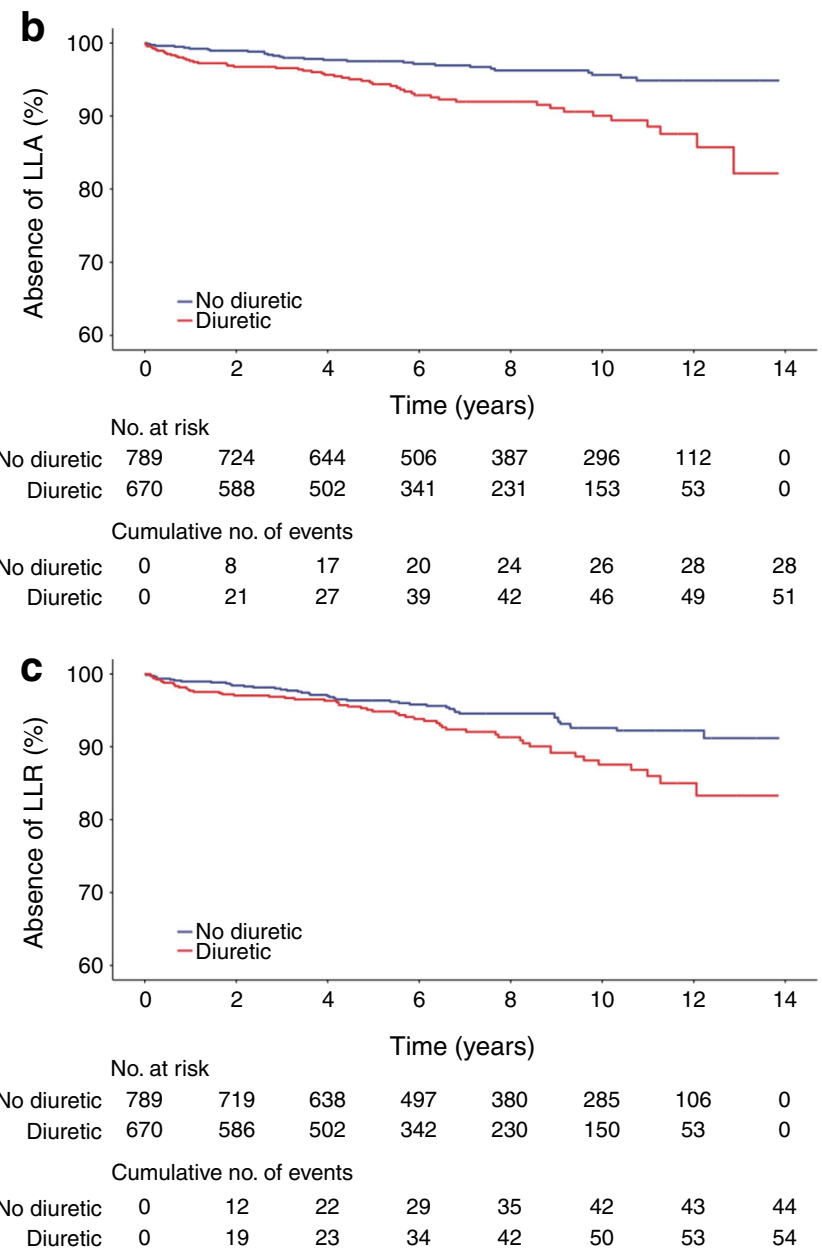

Fig. 1 Kaplan-Meier curves for cumulative-outcome-free survival during follow-up with and without diuretic use: (a) LLE (LLA or LLR); (b) LLA; and (c) LLR. Logrank test, $p<0.001$ for the three graphs

significance (HR 0.87 [0.75, 1.01], $p=0.06$ ) [14]. The Intervention as a Goal in Hypertension Treatment (INSIGHT) trial reported a higher incidence of peripheral vascular disorders with co-amilozide vs nifedipine $(5.3 \%$ vs $3.0 \%, p<0.0001)$ [15]. In line with our results, it could be argued that the trend observed in these trials might be related to a deleterious effect of diuretics rather than a protective action of calcium channel blockers.

While beyond the scope of this observational analysis, we can speculate on the potential underlying mechanism between diuretic exposure and LLE. The most likely mechanism that could explain our results is the extracellular volume depletion induced by diuretics. Indeed, hypovolaemia and dehydration could decrease lower extremity perfusion, trigger haemodynamic decompensation and increase blood viscosity, leading to ischaemia and amputation. A few case series of leg or mesenteric ischaemia related to extracellular volume deficit induced by diuretics were reported in the 1970s [4, 5]. Similarly, Tzoulaki et al showed that increased haematocrit, a marker of blood volume, was significantly associated with incident peripheral artery disease events [16]. Nonetheless, diuretic-induced volume depletion is not similar between the different classes of diuretic. The volume-depletion effect is much more important with loop than thiazide diuretics. It would have been useful to compare the effect of each class of diuretic to assess this hypervolemia-driven hypothesis. However, such data were not available for our cohort. Another theoretical mechanism may be the intracellular swelling due to diuretic-induced electrolyte changes, which has been shown to contribute to the ischaemic process [17].

Recently, the CANVAS trial showed that LLA occurred about twice as often in participants treated with canagliflozin compared with placebo [6]. Consistent with this result, in a population-based cohort study of 25,258 propensity-matched participants, Udell et al observed a twofold risk of below-knee lower extremity amputation associated with the initiation of SGLT2 inhibitors [18]. Although the mechanisms involved are still unknown, in the light of our results, one potential culprit could be the diuretic-like effect of SGLT2 inhibition. If this side effect is caused by drug-induced hypovolaemia, it should be shared throughout the class. However, whether this risk of amputation occurs singly with canagliflozin or is a class effect remains controversial. Recent observational studies and meta-analyses have reported conflicting results [19-27]. An increased risk of LLA was not observed in participants using empagliflozin in the Empagliflozin Cardiovascular Outcome Event Trial in Type 2 Diabetes Mellitus Patients (EMPA-REG OUTCOME) trial; however, LLAs were identified manually in a post hoc analysis [28]. Nonetheless, in a recent analysis of nationwide registers from Sweden and Denmark including 34,426 propensity-matched participants, use of SGLT2 inhibitors, with only $1 \%$ of canagliflozin users, was associated with a twofold risk of LLA compared with glucagon-like peptide 1 (GLP1) receptor agonists [27]. Although no data are available regarding a potentially different diuretic effect of SGLT2 inhibitor molecules, this could be an explanation of the discrepancy observed for amputation risk in these studies. 
Table 2 Risk of LLE, LLA and LLR during follow-up associated with diuretic use at baseline

\begin{tabular}{|c|c|c|c|c|c|c|}
\hline \multirow[t]{2}{*}{ Adjustment } & \multicolumn{2}{|l|}{ LLE } & \multicolumn{2}{|l|}{ LLA } & \multicolumn{2}{|l|}{ LLR } \\
\hline & $\operatorname{HR}(95 \% \mathrm{CI})^{\mathrm{a}}$ & $p$ & $\mathrm{HR}(95 \% \mathrm{CI})$ & $p$ & $\mathrm{HR}(95 \% \mathrm{CI})$ & $p$ \\
\hline Unadjusted & $2.08(1.49,2.93)$ & $<0.001$ & $2.53(1.61,4.10)$ & $<0.001$ & $1.71(1.15,2.56)$ & 0.01 \\
\hline Adjusted $^{\mathrm{b}}$ & $1.49(1.01,2.19)$ & 0.04 & $2.01(1.14,3.54)$ & 0.02 & $1.05(0.67,1.64)$ & 0.84 \\
\hline $\begin{array}{l}\text { Death as competing } \\
\text { risk }^{\mathrm{c}}\end{array}$ & $1.89(1.35,2.64)$ & $<0.001$ & $2.28(1.44,3.62)$ & $<0.001$ & $1.52(1.02,2.27)$ & 0.04 \\
\hline
\end{tabular}

Number of individuals included in the Cox analyses: 1459 in the unadjusted analysis; 1418 in the adjusted model; and 1459 in the Fine and Gray model

${ }^{\text {a }}$ Subhazard ratio (95\% CI) shown for death as competing risk

${ }^{\mathrm{b}}$ Adjusted for age, diabetes duration, sex, BMI, systolic BP, hypertension, diabetic retinopathy, previous cardiovascular disease, previous amputation, previous PAD, HDL-cholesterol, ACR, eGFR, antiplatelet/anticoagulant, BP-lowering agents, statins, biguanides and insulin use

${ }^{\mathrm{c}}$ Fine and Gray model (competing risk = all-cause death)
This diuresis-driven hypothesis could have been reinforced with a stronger association in participants with PAD at baseline, as observed in CANVAS trial and the study of Udell et al $[6,18]$. However, the number of such participants in our study was too limited to test this hypothesis $(n=73)$ (ESM Table 1). Larger studies are certainly required for further assessment.

Our study had several limitations. Most of the weakness and limitations were related to the nature of the dataset. Indeed, we analysed a prospective observational cohort initially created to assess the genetic determinant of diabetic nephropathy and not a drug effect. Therefore, the lack of details on treatments and the potential residual confounding due to the observational nature of our cohort were the main limitations of our study. First, we assessed only baseline exposure to diuretics, leaving some uncertainty about their introduction or interruption, possibly blurring our results. Similarly, as the index date for drug initiation was not available, we could not assess time-varying hazards and drug effects associated with treatment duration. Lack of data on date of initiation and interruption of diuretics was a major limitation of our results. Second, because of its observational design, our study did not allow any direct exploration of a causal relationship between diuretic use and LLE. We are aware that the most relevant method for examining such a deleterious effect of diuretics would be to focus on a new user cohort and compare users of diuretics with users of other non-diuretic BP-lowering medications to minimise selection bias and immortal time bias. However, despite the limited number of participants, we found a similar, albeit non-significant, trend towards a higher risk of

Table 3 Risk of LLE, LLA and LLR during follow-up associated with diuretic use at baseline in the propensity-score-matched cohorts

\begin{tabular}{|c|c|c|c|c|c|}
\hline \multicolumn{2}{|l|}{ LLE } & \multicolumn{2}{|l|}{ LLA } & \multicolumn{2}{|l|}{ LLR } \\
\hline HR (95\% CI) & $p$ & HR $(95 \% \mathrm{CI})$ & $p$ & HR (95\% CI) & $p$ \\
\hline $1.53(1.05,2.24)$ & 0.03 & $1.98(1.15,3.40)$ & 0.01 & $1.11(0.72,1.71)$ & 0.65 \\
\hline
\end{tabular}

LLE with RAAS-blocker use ( $p=0.07$; ESM Table 1). The SURDIAGENE cohort was also taken from a single French diabetes department and may not be representative of all populations with type 2 diabetes. However, as the decision about amputation is not standardised practice and is probably dissimilar between different centres, it could lead to bias in such a study, so the single-centre design (with homogeneous amputation practice) for our cohort could be a point of strength.

Our findings can be generalised only for white people with type 2 diabetes. Moreover, measures to assess volaemic status were not available in this cohort to test our volaemia hypothesis.

Finally, despite multiple adjustments, we cannot avoid the possibility of residual confounding from both potential unmeasured confounders and residual confounding in measured confounders. As the baseline characteristics of participants clearly showed a higher burden of complications, the risk of confounding by indication was high. As a sensitivity analysis, we analysed the association between outcomes and diuretic use after propensity-score adjustment and propensity-scorematched sample with well-balanced covariates across both groups. However, despite all the adjustments, we cannot exclude that our findings are more the consequence of an inadvertent pre-selection of participants than a drug effect.

Conclusions In conclusion, we consolidate and expand previous findings showing that diuretics are associated with an increased risk of LLA and LLR in participants with type 2 diabetes. Our results provide a potential explanation for the increased risk of LLA observed with canagliflozin. However, the SURDIAGENE cohort was not designed to assess the impact of drugs on outcomes and our results should be interpreted with caution; further analyses are needed for confirmation.

Acknowledgements We thank: all participants included and followed-up in the cohort study for their kind participation in this research; the nurses 
and technicians who contributed to this clinical study; and E. Gand for her help with data management.

The independent adjudication committee for the SURDIAGENE study includes: J. M. Halimi (Chairman Tours, France), G. Ducrocq (Paris, France), R. Roussel (Paris, France), P. Llatty (Poitiers, France), V. Rigalleau (Bordeaux, France), C. Hulin (Poitiers, France), D. Montaigne (Lille, France) and P. Zaoui (Grenoble, France).

Some of the data were presented as an abstract at the EASD meeting in 2018 (session 7, abstract 12).

Data availability The datasets analysed during the current study are not publicly available because of intellectual property considerations and continuing analyses by the study investigators; they are available from the corresponding author on reasonable request.

Funding The SURDIAGENE study was supported by grants from the French Ministry of Health (Programme Hospitalier de Recherche Clinique [PHRC]-Poitiers 2004) and the Association Française des Diabetiques (AFD) (Research Grant 2003). The sponsors had no role in the: design or conduct of the study; collection, management, analysis or interpretation of the data; preparation, review or approval of the manuscript; or decision to submit the manuscript for publication.

Duality of interest LP reports grants, personal fees and non-financial support from Novo Nordisk and Sanofi, personal fees and non-financial support from Eli Lilly and non-financial support from Servier. RR reports grants, personal fees and non-financial support from Sanofi, personal fees and non-financial support from MSD, grants from Amgen, personal fees from Physiogenex, AstraZeneca, Janssen, Eli Lilly, Abbott, Medtronic, Novo Nordisk and Servier and grants from Novo Nordisk. AB reports non-financial support from Sanofi, Novo Nordisk and Elivie. MM reports personal fees from Abbott, Intarcia, Eli Lilly, MSD, Novo Nordisk, Sanofi and Servier, grants from Novo Nordisk, Sanofi, Servier, MSD and Novartis and non-financial support from Novo Nordisk. KM reports personal fees and non-financial support from Novo Nordisk and Sanofi and non-financial support from VitalAir. SH reports personal fees and non-financial support from AstraZeneca, Bristol-Myers Squibb, Janssen, MSD and Sanofi, personal fees from Abbott, Boehringer Ingelheim, Eli Lily, Novartis, Novo Nordisk, Servier and Takeda. All support was outside the submitted work and modest. All other authors declare that there is no duality of interest associated with their contribution to this manuscript.

Contribution statement LP had full access to all the data in the study and takes responsibility for the integrity of the data and the accuracy of the data analysis. LP, RR, KM and SH made substantial contributions to conception and design of the study. All authors made substantial contributions to acquisition, analysis or interpretation of data and gave final approval of the version to be published. LP and RR drafted the manuscript and all the authors made critical revision of the manuscript for important intellectual content.

Publisher's note Springer Nature remains neutral with regard to jurisdictional claims in published maps and institutional affiliations.

\section{References}

1. van Houtum WH, Lavery LA, Harkless LB (1996) The impact of diabetes-related lower-extremity amputations in The Netherlands. J Diabetes Complications 10(6):325-330. https://doi.org/10.1016/ 1056-8727(95)00088-7

2. Davis WA, Norman PE, Bruce DG, Davis TM (2006) Predictors, consequences and costs of diabetes-related lower extremity amputation complicating type 2 diabetes: the Fremantle Diabetes Study. Diabetologia 49(11):2634-2641. https://doi.org/10.1007/ s00125-006-0431-0

3. Martinez-De Jesus FR (2010) A checklist system to score healing progress of diabetic foot ulcers. Int J Low Extrem Wounds 9(2):74 83. https://doi.org/10.1177/1534734610371594

4. Sharefkin JB, Silen W (1974) Diuretic agents: inciting factor in nonocclusive mesenteric infarction? JAMA 229(11):1451-1453. https://doi.org/10.1001/jama.1974.03230490039020

5. O'Rourke DA, Hede JE (1978) Reversible leg ischaemia due to diuretics. Br Med J 1(6120):1114. https://doi.org/10.1136/bmj.1. 6120.1114

6. Neal B, Perkovic V, Mahaffey KW et al (2017) Canagliflozin and cardiovascular and renal events in type 2 diabetes. $\mathrm{N}$ Engl $\mathrm{J}$ Med 377(7):644-657. https://doi.org/10.1056/NEJMoa1611925

7. Erkens JA, Klungel OH, Stolk RP, Spoelstra JA, Grobbee DE, Leufkens HG (2004) Antihypertensive drug therapy and the risk of lower extremity amputations in pharmacologically treated type 2 diabetes patients. Pharmacoepidemiol Drug Saf 13(3):139-146. https://doi.org/10.1002/pds.932

8. Gary T, Belaj K, Hafner F et al (2015) Graz critical limb ischemia score: a risk score for critical limb ischemia in peripheral arterial occlusive disease. Medicine (Baltimore) 94(27):e1054. https://doi. org/10.1097/MD.0000000000001054

9. Hadjadj S, Fumeron F, Roussel R et al (2008) Prognostic value of the insertion/deletion polymorphism of the ACE gene in type 2 diabetic subjects: results from the Non-insulin-dependent Diabetes, Hypertension, Microalbuminuria or Proteinuria, Cardiovascular Events, and Ramipril (DIABHYCAR), Diabete de type 2, Nephropathie et Genetique (DIAB2NEPHROGENE), and Survie, Diabete de type 2 et Genetique (SURDIAGENE) studies. Diabetes Care 31(9):1847-1852. https://doi.org/10.2337/dc072079

10. Levey AS, Stevens LA, Schmid CH et al (2009) A new equation to estimate glomerular filtration rate. Ann Intern Med 150(9):604 612. https://doi.org/10.7326/0003-4819-150-9-200905050-00006

11. Fine JP (2001) Regression modeling of competing crude failure probabilities. Biostatistics 2(1):85-97. https://doi.org/10.1093/ biostatistics/2.1.85

12. Ebrahimian TG, Tamarat R, Clergue M, Duriez M, Levy BI, Silvestre JS (2005) Dual effect of angiotensin-converting enzyme inhibition on angiogenesis in type 1 diabetic mice. Arterioscler Thromb Vasc Biol 25(1):65-70. https://doi.org/10.1161/01.ATV. 0000149377.90852.d8

13. Austin PC (2011) An introduction to propensity score methods for reducing the effects of confounding in observational studies. Multivariate Behav Res 46(3):399-424. https://doi.org/10.1080/ 00273171.2011 .568786

14. ALLHAT Officers and Coordinators for the ALLHAT Collaborative Research Group (2002) Major outcomes in high-risk hypertensive patients randomized to angiotensin-converting enzyme inhibitor or calcium channel blocker vs diuretic: the Antihypertensive and Lipid-Lowering Treatment to Prevent Heart Attack Trial (ALLHAT). JAMA 288(23):2981-2997. https://doi.org/10.1001/ jama.288.23.2981

15. Brown MJ, Palmer CR, Castaigne A et al (2000) Morbidity and mortality in patients randomised to double-blind treatment with a long-acting calcium-channel blocker or diuretic in the International Nifedipine GITS study: Intervention as a Goal in Hypertension Treatment (INSIGHT). Lancet 356(9227):366-372. https://doi. org/10.1016/S0140-6736(00)02527-7

16. Tzoulaki I, Murray GD, Lee AJ, Rumley A, Lowe GD, Fowkes FG (2007) Inflammatory, haemostatic, and rheological markers for incident peripheral arterial disease: Edinburgh Artery Study. Eur Heart J 28(3):354-362. https://doi.org/10.1093/eurheartj/ehl441 
17. Leaf A (1973) Cell swelling. A factor in ischemic tissue injury. Circulation 48(3):455-458. https://doi.org/10.1161/01.CIR.48.3. 455

18. Udell JA, Yuan Z, Rush T, Sicignano NM, Galitz M, Rosenthal N (2018) Cardiovascular outcomes and risks after initiation of a sodium glucose cotransporter 2 inhibitor: results from the EASEL population-based cohort study (Evidence for Cardiovascular Outcomes With Sodium Glucose Cotransporter 2 Inhibitors in the Real World). Circulation 137(14):1450-1459. https://doi.org/10. 1161/CIRCULATIONAHA.117.031227

19. Fadini GP, Avogaro A (2017) SGTL2 inhibitors and amputations in the US FDA Adverse Event Reporting System. Lancet Diabetes Endocrinol 5(9):680-681. https://doi.org/10.1016/S2213-8587(17) 30257-7

20. Khouri C, Cracowski JL, Roustit M (2018) SGLT-2 inhibitors and the risk of lower-limb amputation: is this a class effect? Diabetes Obes Metab 20(6):1531-1534 .https://doi.org/10.1111/dom.13255

21. Adimadhyam S, Lee TA, Calip GS, Smith Marsh DE, Layden BT, Schumock GT (2018) Risk of amputations associated with SGLT2 inhibitors compared to DPP-4 inhibitors: a propensity-matched cohort study. Diabetes Obes Metab 20(12):2792-2799. https://doi. org/10.1111/dom.13459

22. Yuan Z, DeFalco FJ, Ryan PB et al (2018) Risk of lower extremity amputations in people with type 2 diabetes mellitus treated with sodium-glucose co-transporter-2 inhibitors in the USA: A retrospective cohort study. Diabetes Obes Metab 20(3):582-589. https://doi.org/10.1111/dom.13115
23. Radholm K, Wu JH, Wong MG et al (2018) Effects of sodiumglucose cotransporter-2 inhibitors on cardiovascular disease, death and safety outcomes in type 2 diabetes - A systematic review. Diabetes Res Clin Pract 140:118-128. https://doi.org/10.1016/j. diabres.2018.03.027

24. Dawwas GK, Smith SM, Park H (2019) Cardiovascular outcomes of sodium glucose cotransporter-2 inhibitors in patients with type 2 diabetes. Diabetes Obes Metab 21(1):28-36. https://doi.org/10. 1111/dom.13477

25. Chang HY, Singh S, Mansour O, Baksh S, Alexander GC (2018) Association between sodium-glucose cotransporter 2 inhibitors and lower extremity amputation among patients with type 2 diabetes. JAMA Intern Med 178(9):1190-1198. https://doi.org/10.1001/ jamainternmed.2018.3034

26. Ryan PB, Buse JB, Schuemie MJ et al (2018) Comparative effectiveness of canagliflozin, SGLT2 inhibitors and non-SGLT2 inhibitors on the risk of hospitalization for heart failure and amputation in patients with type 2 diabetes mellitus: A real-world meta-analysis of 4 observational databases (OBSERVE-4D). Diabetes Obes Metab 20(11):2585-2597. https://doi.org/10.1111/dom.13424

27. Ueda P, Svanstrom H, Melbye M et al (2018) Sodium glucose cotransporter 2 inhibitors and risk of serious adverse events: nationwide register based cohort study. BMJ 363:k4365

28. Inzucchi SE, Iliev H, Pfarr E, Zinman B (2018) Empagliflozin and assessment of lower-limb amputations in the EMPA-REG OUTCOME trial. Diabetes Care 41(1):e4-e5. https://doi.org/10. 2337/dc17-1551 